Lapurdum

Euskal ikerketen aldizkaria | Revue d'études basques |

Revista de estudios vascos | Basque studies review

$4 \mid 1999$

Numéro IV

\title{
Bernard Lehete euskaldun mezenas bat Bordelen XVI. mendean
}

\section{Patri Urkizu}

\section{OpenEdition}

\section{Journals}

Édition électronique

URL : http://journals.openedition.org/lapurdum/1546

DOI : 10.4000/lapurdum.1546

ISSN : 1965-0655

Éditeur

IKER

Édition imprimée

Date de publication : 1 octobre 1999

Pagination : 107-114

ISBN : 2-84127-156-0

ISSN : $1273-3830$

Référence électronique

Patri Urkizu, « Bernard Lehete euskaldun mezenas bat Bordelen XVI. mendean », Lapurdum [Linean], 4 । 1999, Sarean emana----an 01 avril 2010, kontsultatu 29 janvier 2020. URL : http://

journals.openedition.org/lapurdum/1546; DOI : 10.4000/lapurdum.1546 


\section{Patri URKIZU}

\section{BERNARD LEHETE EUSKALDUN MEZENAS BAT BORDELEN XVI. MENDEAN}

Jean Haritschelhar irakasle agurgarriari nere eskerrik beroenak adieraziz hasi nahi nuke egungo solasa, berari esker eman bainituen lehen urratsak Bordeleko Unibertsitatean, Bordeaux III-en, egun Michel de Montaigne deituan, hain zuzen, eta bertan hiru urte benetan probetxugarri eta ahantzezinak igaro.

Hori dela eta, bururatu zait lehen euskal liburu inprimatua, alegia, Beñat Detxeparek' idatzitako Linguae Vasconum primitiae, Bordelen 1545ean moldiztegiratua izan zela eta Bernard Leheteri eskainirik dagoenaren aitzakiaz, honen eta garai hartan Bordelen bizi izan ziren zenbait poeten inguruan mintzatzea, hots, Errenazimenduko poesiaz aritzea.

Ez dakigun arren gauza handirik Bernard Leheteren bizitzaz, egia esan, badakigu zertxobait bederen. Adibidez, Alfred Lassus-ek dioskunez ${ }^{2}$, Sarako familia nobleetarik zela, oraindik bertako etxe eder batek izen bera daramalarik. M.A. Pegg-ek ${ }^{3}$ emaniko ohar batez jakin, badakigu, berriz, 1514. urtean Tolosan, hau da Frantziako Toulouse-n, ikasten ari zela eta bertan lecteur-irakurle- gisa aritu zela 1516an. Urte batzuek geroxeago abokatu zela Guyenne-ko amiralgoan (1524-1529) eta abokatu jenerala legez lanean hasi zela 1529, kargu berean jarraikiz 1560a arte, azken hatsa 1562 an eman zuelarik Bordelen.

Suertatu zaigu ere bere autografo dotorea ikustea hala nola bere seme Joanesena Bordeleko herri liburutegian, eta bidenabar Girondako Artxiboetan ${ }^{4}$ aurki daitezke beste zenbait albiste bere bizitzaz. Hala nola 1530.eko abuztuaren 22 an hartu zuela bere lan kargua, aurrekoa, Thomas Cousinier Provence-ko Parlamentuko lehendakari bezala hautatua izan zelako ${ }^{5}$.

Halaberetsu badakigu bere seme Joanesi utzi ziola kargua, 1554eko martxoaren 4ean, Fontainebleau-n sinatu agiriaren arabera, baina honek ezin izan zuela karguaz arduratu $1560 \mathrm{a}$ arte gaztetxo zelako oraindik. Halere, 1564an gure Bernard Laheteren semea Joanes prokuradore jenerala zen, eta eliza erreformatuko Guilloche kontseilariaren lehengusua. Hau dela eta katoliko zenbaitek salatu egin zuten ${ }^{6}$ haren jauregia erreformatu iraultzaileen babeslekua bilakatua zelako, eta istilu hanitz pairatu zituen kausa honengatik. dena den, Parisen hil zenean 1572an Bordeleko Parlamentu osoak parte hartu zuen hileta elizkizunetan, zeinek adierazten duen argi eta garbi zein estimu handitan zeukaten bordeleko hiritarrek gure Joanes ${ }^{7}$.

Hemen interesatzen zaiguna, ordea, bere aita Bernard da, zeren honek bildu zituen, bederen, bi poeta handiren laudorioak. Bata Beñat Detxepare, lehen euskal liburu inprimatuaren egilearen partetik, eta bestea Eustorg de Beaulieu garaian poeta ospetsuaren aldetik. 
Rregueren aduocatu videzco eta noblea
ri virtlute eta honguciez complitu pari
bere iaun eta jabe Bernard Leheteriber
uard echeparecoac haren cerbitzari chi
piac gogo honez goray ncibague et a of Iagarri Ceren bafcoac baitira abil animos eta gentil etă hetan içan baita eta baita fciencia gucietan letrratu hathdiric miraz nago iauna nola batere ezten affay atu bere lengoage propriaren fauoretan heufcaraz cerbait obra eguitera eta foributan imeitera ceren ladin publi cal mundu gucietara berce lengoagiac beçala hay $n$ fcti batzeco hondela. Eta cauía honegatic gueldit zenda a* bataturic eceyn reputacione vague etaberce nacione oroc vife date ecin denfere feriba dayteycla lengoage hartan nolaberce oroc baitu te fcribatzen beryan Eta ceren oray quc iauna noble et naturazcoac beçala bay tuçu eftimatzen goratzen cta ohoratzen heufcara gu. ri neure iaun eta iabia beçala jgortendarauritzut heu fcarazco copblabatzuene ignoranciaren araura eguin nac. Ceren iauna hayec iqhuffiric eca corregituric pla zer duçun beçala irudi baçautzu imprimieraci diça: çun eta çure efcutic oroc dugum ioya ederra Impria mitu ric heufcara orano içan eztena et a cure hat fe ho. netic dad in aitzinerat augmenta continua eta publica mundu gucietar a cta balcoec bercec becala duten be re lengoagian feribuz cerbait doft ina eta plazer har. ceco folaz eguiteco cantatzeco eta denbora igaraiteco materia et a ginendirenec gue 10 duten caufa oboro ha rea aban ̧̧atzeco eta obligatu guiren guciac ge y ncoari A $i j$ 
Honelaxe hasten du Detxeparek, justuki, hain ezaguna den bere eskaintza :

Erregueren advocatu videzco eta nobleari virtute eta hon guciez complituya$r i$ bere iaun eta iabe Bernard Leheteri bernard echeparecoac haren cerbitzari chipiac gogo honez gorayncy baque eta osagarri...

Aurreraxeago euskalzaletzat jotzen du, noski, alegia, euskara ohoratu duela abokatu bordelesak, seguruen inprimatze baimena lortzeko bitartekari izanen baitzen, hitzokin :

Eta ceren oray çuc iauna noble eta naturazcoac beçala baytuçu estimatzen goratzen eta ohoratzen heuscara çuri neure iaun eta iabia beçala igorten darauritzut heuscarazco copla batzu..

Bukaeraldean koplen helburua honelaxe agertzen digularik :

... eta bascoec bercec beçala duten bere lengoagian scribuz cerbait doctrina eta plazer harceco solas eguiteco cantatzeco eta denbora igaraiteco materia...

Nabari denez, musikak eta kantak berekin dakarren plazera ere ez zegoen coplac idaztean egileak zituen helburuetarik at. Ez naiz honetaz gehiago mintzatuko aski baitira aritu ni baino lehenago hainbat eta hainbat kritikari, tartean Haritschelhar jauna bera. Mintza nadin, beraz, Eustorg de Bealieu, honek ez hitz lauz baizik bertsotan, aitzinago emango dugun ehun bertsolerroko poema eskaini zionez gure Bernard Laheteri.

Eustorg de Beaulieu, Beaulieu-sur-Ménoire-n jaio zen 1495ean. Bere ama Jeanne de Bosredon deitzen zen, eta umezurtz gelditu zen oso gazte, zaindariak diru guztiak xahutu zizkiolarik. Bordeleko parlamentuaren aitzinean salatu zuen, bai abokatuari bai eta epaileei bertsoak zuzendu zizkielarik, 1529an justizia lortuz.

Urte honetan plazaratu zuen Bordelen Les gestes des solliciteurs poema liburua, non apez bezala, prestre, ageri zaigun eta non tribunaletako hainbat jende kritikatzen dituen.

Garai honetan Tulle-n bizi zclarik oso ospetsua zen teatrogileen artean bere ausardia eta literatur talentuak zirela eta, zenbait antzerki idatzi zuelarik honako tituluak zutenak : Histoire de l'enfant prodigue, Le murmure et fin de Choré, Dathan et Abiron...

Esan bezala Lehetek babestu zuen, eta honen alaba Margaritaren maisu ere izan zen ${ }^{8}$. Gero François de La Tour, Turenne-ko bizkontearen zerbitzura igaro zen, eta 1536an Lyon-era Charles d'Estaing-endako gomendiozko gutun batekin. Etxerik hoberenetan errezibitu zuten, Hélène de Gondi-ren irakasle izan zen eta Lyon-en 1537an Divers rapportz, contenant plusieurs rondeaux, dixains et ballades", argitaratu zuen, non amodiozko eta laudoriozko kantak ematen zituen, hala nola andere gorputzaren zazpi soin-armei buruzko bertsoak, askeegitzat jo zirenak.

Eliza erreformatuarekin harremanetan zen, eta beraz, bai Erasmoren ohoretan bai eta Margarita Nafarroakoren alde bertsoak idatzi zituen, eta Mélanchton-en lantxo bat itzuli zuen. !537ko maiatzaren lehenean Genève-n babestu zen, bertan urte bat emanez, ondoren Lausanne-ra teologia ikastera joan zelarik. 1540an Bernan zen, eta artzain hautatu zuten Thierrens, Vaud-eko lurraldean, bertan 
zazpi urtez biziz, eta bai polemika lanak bai kanta ezpiritualak idatziz, hala nola San Pabloren gutunak eta Psalmoak itzuliz. Garai honetan, hots, 1546. urtean plazaratu zuen Chrestienne resjouissance ${ }^{10}$. Titulu luzean dioen bezala, apez, musikari eta organistatzat jotzen zuen bere burua. Liburu honetan 160 kantu ageri dira, zeinen hitzak eta musika sortzeaz harro sentitzen den. Halere Paul Meyer ", kritikariak dioenez, ez ziren hain originalak, garaian modan zirenetik imitatu baitzituen bai bertsoak, - batzuetan hitzez-hitz kopiatuz, bai eta doinuak. Zenbait kantatan aitasaindua, fraideak eta peregrinazioak kritikatzen ditu oso modu bizian. Eustorg Bâlen hil zen 1552eko urtarrilaren 8an.

Hona Eustorg de Beaulieu-k Bernard Leheteri eskainitako poema :

Jadis envoyée de par l'Aucteur à scientifique \& Tres-prudent Seigneur. Monsieur Maistre Bernard de Lahet, Advocat du Roy en sa Court de Parlement à Bordeaulx.

Combien que Craincte en t'escripvant s'avance

De m'appeller Fol, plain d'oultrecuydance,

Espoir me poulse \& me dict : Tu fais mal

4 Si tu te craintz q'ung cueur Noble \& Loyal,

Tant enrichi de vertus à souhait

Comme celuy de Bernard de Lahet, Aye en desdain la teneur de ta lettre,

8 Quoy qu'elle soit en rude stile \& metr:;

Et (que plus est) qu'il soit en dignité

(Non sans merite) \& grande auctorité,

Car ja pour ce ne t'en tiendra à mespris,

12 Et n'en seras aulcunement repris,

Joinct que les Biens, \& les Honneurs du monde

N'ont rien changé ses meurs \& sa faconde,

Et ne fut onq plus doulx \& liberal

16 (Avant qu'il feust Advocat general)

Que maintenant, sellon ceulx qui le hantent,

Et à toute heure au pres de lui frequentent.

Or ce voyant, j'ay dict à Crainete \& Peur

20 Qu'en despit d'eulx je prendrois le labeur

D'ung mot t'escripre en lourde Rymasseure,

Voyant que Espoir (que maintz nourrit) m'asseure,

le suppliant pardonner (toutesfois)

24 Ma folle audace, au moins pour ceste fois, Combien que croy que trop ingrat seroye

S'à tout le moins ce coup ne t'escripvoye, Veu les proffitz, les biens \& les honneurs

28 Que j'ay receupz de toy en lieux plusieurs, Sans que jamais te feisse aulcuns services Dignes en rien de tant de benefices ;

Entre lesquelz j'ose bien reciter 
32 Que je t'ay veu pour moy solliciter Et'veoir mon sac, Puis en faire sermon Au bon seigneur Monsieur de sainct Symon Qu'en mon proces lut commis Rapporteur,

36 Dont (grace a Dieu) j'euz par son rapport heur, Car me garda sans faveur ne malice Tresbien mon Droict en forme de Justice. Mais les Raisons de droict que tu me feiz,

40 A sçavoir mon si j'en feiz mes proffitz: Je te prometz que ouy, tant que de choses Ne d'aultre piece en mon dict Sac enclose; Et y parut, dont j'en mercie Dieu,

44 Qui me guida te trouver en ce licu. Tant d'aultres Biens j'euz par ton acointance, Et de Faveur, que je n'auroys puissance Le desservir de cent ans ne de plus,

48 Mais Dieu (qui peult) parfaira le surplus. Je pense fort si ton cueur se delecte (Cornme j'ay veu) au Jeu de I'Espinete? Et s'aymes tant le doulx bruyt Organique

52 Cornme aultres fois, \& la doulce Musique Où je t'ay veu prendre tant de plaisir Que bien souvent, ains que t'aller gesir (Pour de soucy \& peyne estre delivre),

56 Si tu trouvois de Musique aulcung Livre, Toy, Blaise, \& moy, chantions jusque à my nuyct, Sçachant que Dueil ne proffite mais nuict? Et, pour plus estre à la Musique enclin,

60 Tu t'aconctoys de Clement Jennequin Et d'aultres maintz, toutz gens d'experience, Et où gisoit Musicalle science. C'estoit en l'An mil cinq cens vingt \& neuf,

64 Q'ung pain d'ung liard n'estoit plus gros q'ung ceuf; Voire ne fut cent ans avant Cherté Telle que alors, ne si grand Paovreté. Ce non obstant, à tes Coustz \& Despens

68 Tu tins Maison ouverte à plusieurs Gens. $J$ 'en suis Tesmoing qui ne le puis nyer ; Pareillement maistre François Cornier Et maistre Aubert du Pré, aussi la Chausse

72 Et aultres maintz ayant part à la Saulse. Je Ry souvent lors que je considere Le passetemps que tu euz d'ung petit Heere Que j'achaptay pour de ça m'en venir, 
76 Du quel ung jour (s'il t'en veult souvenir)

Je euz belle peur à cause d'une Bulle

Ou d'ung Papier en forme de Cedulle

Que quelque Gueux vint à ton huys poser,

80 En declairant qu'il venoit se opposer

A mon marché \& achapt du dict Heere,

Lequel Rapport, certes, ne me pleust guere,

Et feuz long temps (comme sqais) sans respit,

84 Fantasié \& tout plain de despit.

Mais peu apres le Cueur si me alla dire

Que ce n'estoit q'ung marché faict pour rire,

Et que à tes gens (pendant nostre Repas)

88 L'avoys chargé pour retarder mes Pas,

En me monstrant que encore ma presence

$\mathrm{Ne}$ te faschoit, ne cheuz toy ma despence.

Noble Seigneur, pour resolution,

92 Par cest escript te fais relation

Que pour affin que Ennuy de moy reculle.

Suis à present en la ville de Tulle'

Tu la cognois, Pour ce n'en dy rien plus,

96 Te suppliant que je ne soye exclus

De ta memoire, encore que ne ays veu signe

(En mon endroit) dont de ce je soys digne ;

Et me commande en toutz lieux ton vouloir,

100 Car de ce faire as sur moy le pouvoir.

Aski adierazgarriak direla uste dut ehun bertsolerro hauek, zeren ikusten ditugun Bernard, Eustorg eta Blaise delako bat (nor ote ?) bildurik eta gauerdiraino kantatzen: Toy, Blaise \& moy, chantions jusque à my nuict (57.bl.). Eta Clément Jennequin ${ }^{12}(\mathrm{sic})$, musikari handia lagun zutela. Nabarmen da, bere etxea, mende bat geroago Bertrand d'Etxauzena bezalaxe artisten babesleku izan zela. Tu tins Maison ouverte à plusieurs Gens (68. bl.), dio zehazki laudoriozko koplak, aipatuz François Cornier eta Aubert du Pré ${ }^{13}$ prokuradoreak.

Kantatuko ote zituen Bernard Lehetek bere Bordeleko jauregian euskal kantak Beñat Detxepareren konpainian ? Neretzat ez dago dudarik baietz, zeren baitirudien Lehetek, Cornier-ek eta du Pré-k Bordeleko parlamentuan goi-kargu zirenak lagun talde eder bat osatzen zutela, musikazale amorratua zena, eta zeinen sarao eta saltsetan, antza denez gure lehen poetak noizpait parte hartuko zuen, zalantzarik gabe. Guzti hauek eta beste zenbaitek osatuko zutekeen, ziuras$\mathrm{ki}$, Bordeleko talde humanista eta errenazentista, tamalez gutxi ezagutzen duguna, eta zeinen artean Clément Marot-en ${ }^{14}$ eragina nabarmena den.

Ez litzateke noski gure poetaren koplen iturriak eta nondikakoak, poetikotasun bideak aztertzeko ikerketa lerro galdua Bordeleko errenazimendu garaia zertxobait gehiago arakatzea. Guk hemen urrats bat besterik ez dugu eman, eta 
hurrengo lanek agian baieztatuko dutelakoan iritzi hau, hemen amaitzen dut solas labur hau.

\section{Résumé :}

Cet article est consacré à Bernard Lehete, mécène basque installé à Bordeaux au XVI ${ }^{\circ}$ siècle. Originaire d'une famille noble de Sare, on sait qu'il était étudiant en 1514 à Toulouse, qu'il fut avocat général à Bordeaux où il mourut en 1562. Dechepare, auteur du premier livre basque, publié en 1545, le remercie d'avoir élevé et honoré la langue basque. Ce travail donne aussi le texte peu connu d'une pièce en vers de Eustorg de Beaulieu (1495-1522) à la gloire de Lehet. On peut donc penser que Lehet abrita à Bordeaux un groupe d'artistes et écrivains chez qui l'influence de Marot ne paraît pas négligeable.

Patri URKIZU

(UNED, Madrid) 


\section{Notes}

1. Linguae Vasconum Primitiae per Dominum Bernardum Dechepare Reciorem sancti michaelis veteris. Bordeaux, François Morpain, 1545.

2. Alfred Lassus, "Les maison nobles, infançonnes autres de Sare et leurs occupants", in Jacques Antz et al., 1993, Sare. Ekaina, 179-211.

3. M. A. Pegg (ed.), 1964, Eustorg de Beaulieu, Les Divers Rapports. Genève, Droz, 239.

4. Archives Historiques du Département de la Girande. Paris. Alphonse Picard et fils, 1901, Vol., xxx, 39.

5. Fond 1B, 1287-1538. Archives de la Gironde. Registre d'enregistrement à Bernard de Lahet de l'office d'avocat général, vacant par la promotion de Thomas Cousinier, à l'office de Président du Parlement de Provence. fol. 158.

6. Lettre contre Lahet. Archives Historiques de la Gironde, X, X. 1487-1567. Registre des enregistrements. n.5. fol. $117 \mathrm{v}$.

7. Jean Lahet (1533 - Paris, 1572)

8. Epistre de L'Autheur à noble \& Chrestienne Damoyseile, Marguerite de sainct Simon en Xaintonge, jadis son escollier. Chrestienne resjouissance..., 1546, 200.

9. Les divers rapportz contenantz plusieurs rondeaulx, Dizains, Ballades, Chansons, Epistres, Blasons, Epitaphes, \& aultres ioyeusetez. Le tout compose par M. E. de beaulieu. 1540. On les vend a Paris en la rue neufve nostre Dame a l'escu de France. Par Alain lotrian

10. Chrestienne resjouissance, composée par Eustorg de Beaulieu, jadis prestre, musicien et organiste en la faulce église papiste é depuis, patı la miséricorde de Dieu, miñistre évangelique en la vayu gi isa do J. C. 1546.

11. Revue critique, 5 octubre de 1872

12. Clément Janequin (Châtellerault 1485 ing. - Paris, 1558), musikaria, François de Gondi, Lorraineko kardinalaren eta François de Guise-ko jaunek babestua, hala nola Henri Il.aren konpositorea. 1505az geroztik Bordelen ageri da Lancelot du Fau, Parlamentuko lehendakari eta 1515az gero Luçon-go apezpiku izango zenaren inguruan. 1523an Jean de Foix, Bordeleko artzapezpikuak babestu zuen eta 1525 ean Saint Emilion-en Fronde-ko kalonje izanen da. Bere obra nagusia hitu, lau eta bosta ahotsetara diren berrehun eta hirurogeita hamabost kantek osatzen dute, 1520an dagoeneko ospetsu, eta musika profanoan maisu, jenero guztiak landu zituen, lirikoa, narraziozkoa, erotikoa, teatrala... bere kantak eskuzkribuzeskuzkribu zabaltzen zirelarik Europan barrena, eta batipat 1528an moldiztegiratu La Guerre obraren ondoren. Bere liburu famatuenen artean aurki daitezke : Chansons (1528), Verger de musique (I559).

13. Albert du Pré 1535etik 1549ra doan prokuradore zerrenda batean ageri da (Actes de l'Académie des sciences, belles lettres et arts de Bordeaux, 1869)

14. Henry Guy, Clément Marot et son école. Histoire de la poésie française au XVI siècle. Paris, Champion, 1968 